\title{
Marine-derived fatty acids therapy for stroke: a systematic review
}

\section{Abstract}

With stroke burden increasing rapidly in recent years, the need for therapies that mitigate the acute insult persists ${ }^{(1)}$. Experimental stroke evidence has shown a neuroprotective effect of marine-derived n-3 polyunsaturated fatty acids (PUFAs), resulting in improved functional outcomes ${ }^{(2)}$. However, evidence in humans remains unclear. Therefore, we conducted a systematic review ${ }^{(3)}$ to evaluate the effects of marine-derived n-3 PUFAs on functional outcomes and dependence after stroke. Secondary outcomes included vascularrelated death, recurrence, and adverse events.

We searched the Cochrane Stroke Group trials register and the following databases: Cochrane Central Register of Controlled Trials, MEDLINE, Embase, CINAHL EBSCO, and Web of Science (SCI-EXPANDED, CPCI-S, and BIOSIS). We also handsearched relevant sources and used the Science Citation Index Reference Search. We included randomised controlled trials (RCTs) assessing marine-derived n-3 PUFAs intervention in people with history of stroke and/or transient ischaemic attack. Two authors independently selected trials, extracted data, assessed risk of bias, and evaluated the quality of the evidence. We contacted study authors for clarification and conducted random effects meta-analysis or narrative synthesis, as appropriate. We divided studies by follow-up length (up to three months and more than three months).

Twenty-nine RCTs were included. Of these, nine studies (3,339 participants) provided outcome data with only one study assessing acute stroke (subarachnoid haemorrhage). Doses of marine-derived n-3 PUFAs ranged from 400 to 3,300 mg per day. Functionality was assessed only in two small studies, one in each follow-up category. These trials reported a trend in improvement (RR $0.78,95 \% \mathrm{CI}$ 0.36 to $1.68,40$ participants; and mean difference $7.09,95 \%$ CI -5.16 to $19.34,52$ participants; respectively). However, due to imprecision and small sample size, the quality of the evidence was very low. No studies reported on acute ischaemic stroke.

Our results show that, currently, evidence on the effect of marine-derived n-3 PUFAs on functional outcomes of stroke is limited and of very low quality. Therefore, more RCTs are needed, especially in the acute phase, to determine whether such intervention could improve stroke outcomes.

This abstract is based on a post-peer review version of a Cochrane Review. Upon completion and approval, the final version is expected to be published in the Cochrane Database of Systematic Reviews.

Funding (CGAC): Mexican government (CONACYT-I ${ }^{2} \mathrm{~T}^{2}$ ).

\section{Conflict of Interest}

There is no conflict of interest

\section{References}

1. Feigin VL (2019) Int J Stroke 14, 223-37.

2. Fulton L et al. (2018) Stroke, 49, AWP255

3. Alvarez Campano CG et al. (2017) Cochrane Database Syst Rev (Protocol) 